\title{
UV-A in the NICU: New Technology for an Old Challenge
}

JA Brons MSc, R White MD, MS Rea PhD

\begin{abstract}
:
Healthcare-associated infections (HAls) are a serious concern in the NICU. Alternatives or supplements to manual cleaning are increasingly being explored, including ultraviolet (UV) disinfection technologies. A recently-developed hybrid lighting system technology, designed to provide both visible white light and disinfecting UV-A ( $\lambda$ max $=366 \mathrm{~nm}$ ) radiation, was retrofitted into a hospital newborn intensive care unit (NICU). The UV-A dosing was set to levels calculated to be safe for continuous adult occupation. The results showed that eight-hour exposures of $3 \mathrm{~W} \mathrm{m-2}$ on counter surfaces were effective for suppressing bacteria that commonly cause HAls in the NICU. Although UV-A is not as effective at inactivating viruses as UV-C, it is safe for use while space is occupied, making it a promising technology for consideration in certain areas of the NICU.
\end{abstract}

Keywords: Ultraviolet UV-A germicidal disinfection irradiation; No-touch cleaning; Healthcare-acquired infections (HAIs); Newborn intensive care unit (NICU)

\section{Introduction}

Approximately 1 in 25 patients in the United States contract healthcare-associated infections (HAls).(1) Patients are particularly at risk if the previous patient in that room had an infection.(2, 3) Standard cleaning procedures usually involve the manual application of detergents, and disinfectants.(4) The efficacy of these manual cleaning procedures can vary considerably among hospitals. $(5,6)$ In fact, less than $50 \%$ of the patient room surfaces are properly cleaned.(1)

Given the incomplete effectiveness of manual cleaning, alternative, so-called, no-touch methods have been examined with the expectation that decontamination of room surfaces will improve when the human element has been removed. Among these notouch methods, the efficacy of short-wavelength optical radiation, from ultraviolet (UV) to blue light (100 to $410 \mathrm{~nm}$ ), has been studied.(7-9) Short wavelengths can inactivate pathogens through two main mechanisms, depending upon the wavelength, duration, and amount of optical radiation(10) as well as the type of pathogen. The direct mechanism involves alterations to DNA or RNA following absorption by UV-C so that the pathogen can no longer replicate. The indirect mechanism involves the absorption of UV-C, UV-B, UV-A, or blue light by chromophores inside or outside the pathogen. These radiation-altered chromophores cause secondary reactions, which, like the direct mechanism, can inactivate replication, but more commonly, they produce chemical reactions that disable the virus, bacterium, or fungus. The efficacy of a given UV dose (intensity $x$ duration) against a given pathogen depends upon the presence or absence of a cell wall, the thick- ness of the cell wall, and the type of nucleic acid.(11) Generally, airborne viruses require lower doses for inactivation than bacteria and fungi, by one or two orders of magnitude. COVID-19, for example, is a single-stranded RNA virus with relatively high cell wall transmissivity to UV-C. In contrast, many bacteria and especially fungi, have double-stranded DNA and low cell wall transmissivity to UV-C (e.g., Candida paraposilosis).

UV-C produced by low-pressure discharge lamps ( $\lambda$ max $\approx 254$ $\mathrm{nm}$ ) been used for many decades to inactivate airborne pathogens. More recently, it has been implemented on mobile platforms that move about the hospital room through remote control to disinfect surfaces. The advantage of UV-C technologies for minimizing HAls is that effective dosage can be achieved with short time durations ( $<1$ hour);(12) the disadvantage of UV-C is that the optical radiation must be applied when the hospital room is unoccupied. For UV-A and blue light applications, several hours of Exposure may be needed to reduce pathogen presence effectively, but, depending upon the wavelength and dose, people can occupy the room without harm. It should be emphasized, however, that all UV technologies are line-of-sight technologies. This means that disinfection can only occur from direct irradiation by the UV source. This works well for airborne pathogens, but those pathogens on shadowed surfaces, such as under a cabinet, will not be affected during UV application.

\section{"With regard to human safety, Exposure to UV-A can cause erythema of the skin (reddening). For wavelengths longer than about 350 nm, however, erythemal effects are negligible.(13) Exposure to short wavelengths longer than about $380 \mathrm{~nm}$ can cause permanent damage to the retina; this phenomenon is known as blue light hazard.(14)"}

With regard to human safety, Exposure to UV-A can cause erythema of the skin (reddening). For wavelengths longer than about $350 \mathrm{~nm}$, however, erythemal effects are negligible.(13) Exposure to short wavelengths longer than about $380 \mathrm{~nm}$ can cause permanent damage to the retina; this phenomenon is known as blue light hazard.(14) Unlike erythemal effects, which depend upon irradiance on the skin, the radiance of the source imaged on the

NEONATOLOGY TODAY is interested in publishing manuscripts from Neonatologists, Fellows, NNPs and those involved in caring for neonates on case studies, research results, hospital news, meeting announcements, and other pertinent topics.

Please submit your manuscript to: LomaLindaPublishingCompany@gmail.com 
retina must be minimized to avoid the blue light hazard, $(14,15)$ So unless the source is extremely bright, little damage to the retina will occur from blue light exposure. According to industry standard safety recommendations(16) then, exposures to UV-A wavelengths between about 350 and $380 \mathrm{~nm}$ can be considered safe for humans at doses that would still be effective for reducing HAI pathogens. Safe exposures of UV-A for infants has not, however, been defined.

The present field study was designed to evaluate a hybrid lighting technology that could provide both visible white light and UV-A optical radiation. The site for the study was a recently-built newborn intensive care unit (NICU). The researchers assessed the efficacy of UV-A exposure for mitigating pathogens found in this unit. Occupant exposure safety was implemented by applying published safety limits(16) for UV-A exposures and confirmed through radiometric measurement. For more information about this study, see Brons et al.(17)

\section{Study Site}

This field study was conducted in the NICU at Memorial Beacon Children's Hospital in South Bend, Indiana, USA. This 39-bed NICU provides an advanced level of care for babies born prematurely or with a critical illness. The facility was designed and built in 2017 to minimize an institutional appearance. For example, rather than caring for multiple babies in one ward, premature babies have private patient rooms (Figure 1). These patient rooms include a private lounge, sleeping, and bathing facilities for each family. Each patient room has a separate sink and counter surface for families and medical staff. The sink and counter area in the patient rooms were the primary focus for assessing UV-A mitigation of pathogens because these high-touch areas are most likely to contain HAls. The study was conducted in six of the NICU patient rooms (Figure 1).

\section{Cleaning}

Nurses work 12-hour shifts, either $07: 00$ to $19: 00$ or 19:00 to 07:00. At the start of each shift (morning and night), nurses clean the sink and counters (PDI Super Sani-cloth germicidal disposable wipes). Once a day, environmental cleaning crews attempt to sanitize the many surfaces in each patient room, including the sink area (Diversey Oxivir 1 Wipes); environmental cleaning staff are not responsible for cleaning the counters.

\section{"Each patient room has a separate sink and counter surface for families and medical staff. The sink and counter area in the patient rooms were the primary focus for assessing UV-A mitigation of pathogens because these high-touch areas are most likely to contain HAls."}

\section{Methodology}

\section{Hybrid Luminaires}

The hybrid luminaires ("Lumination" LBU 22 Disinfection Series D-Light, manufactured by GE Current, a Daintree company) were surface mounted in the patient rooms above the counter and sink areas after the existing luminaires in that area were removed. The hybrid luminaires had two circuits that could be operated independently (Figure 2). White light was provided by conventional light-emitting diodes (LEDs) controlled by the occupant using a dimmable wall switch. The UV-A LEDs were controlled by the manufacturer on a separate circuit using a remotely programmed time clock; the UV-A circuit was set to operate on Wednesdays and Thursdays for 8 hours (09:30 to 17:30). The spectral power distributions (SPDs) of the two luminaire channels are shown in Figure 3. The hybrid luminaires produced a diffuse intensity distribution.
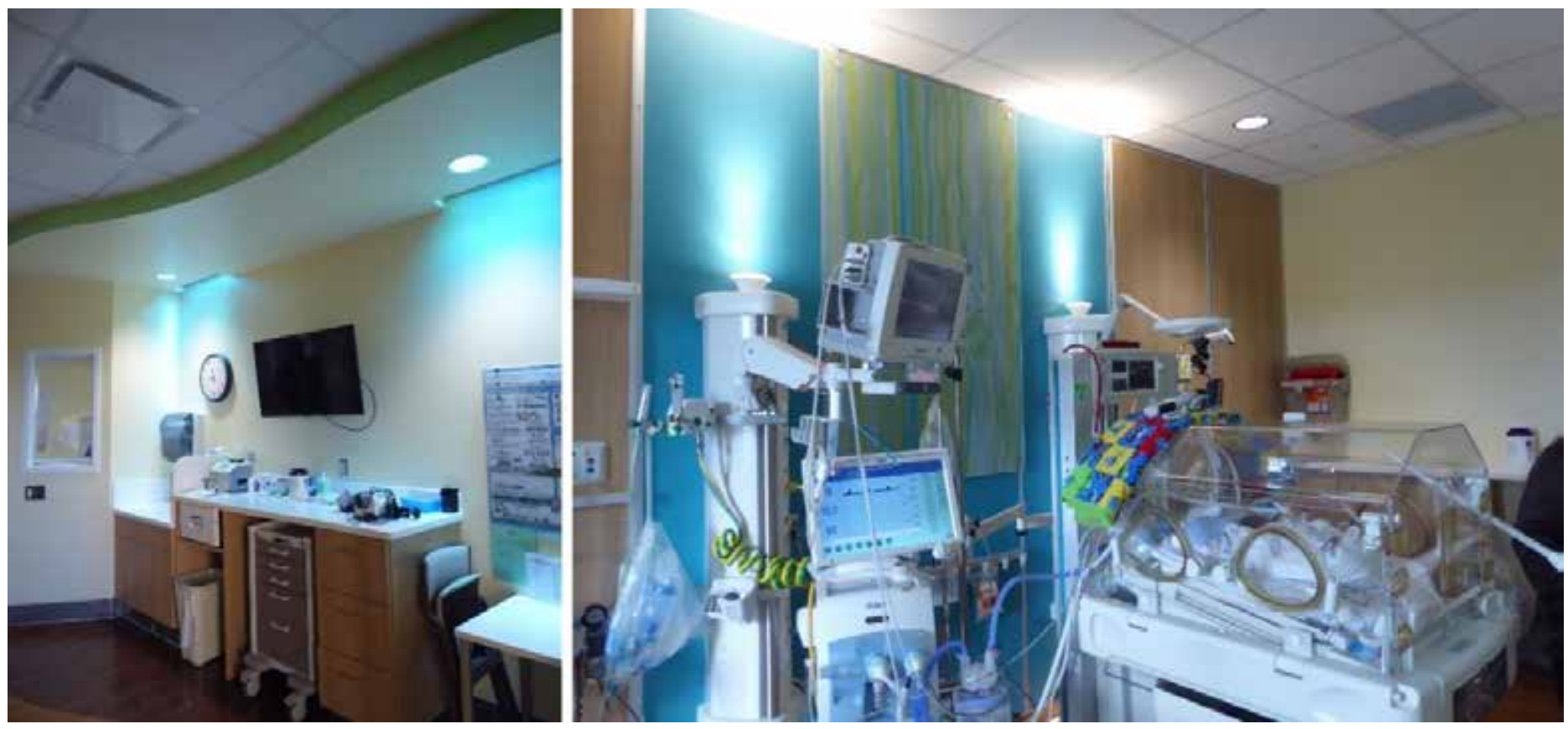

Figure 1. Typical patient room sink and counter, before retrofit with a hybrid lighting system; each patient room serves one family and their infant(s). 

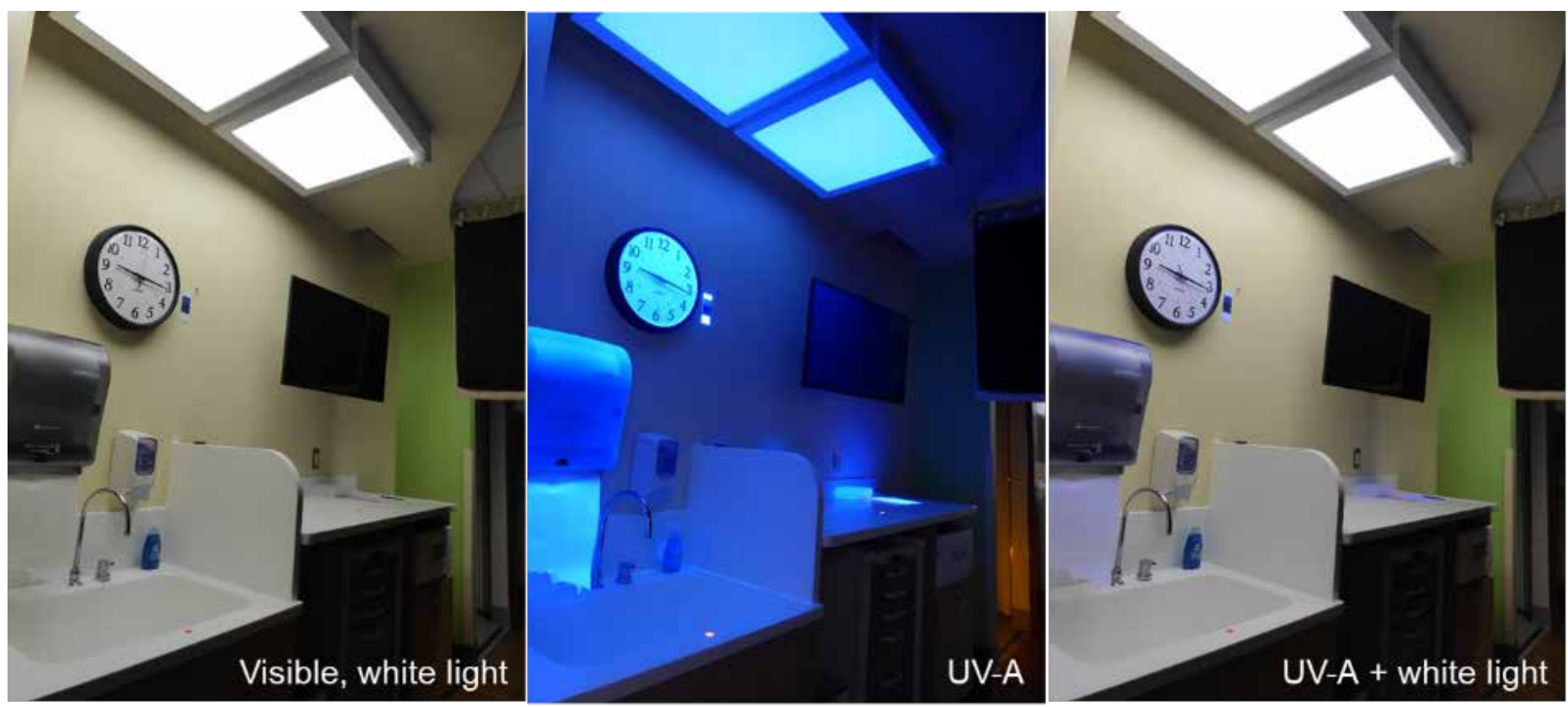

Figure 2. Typical sink and counter space with the hybrid lighting system, providing white light (left) UV-A (center) and both (right). The UV-A and the white light could be energized with separate circuits.

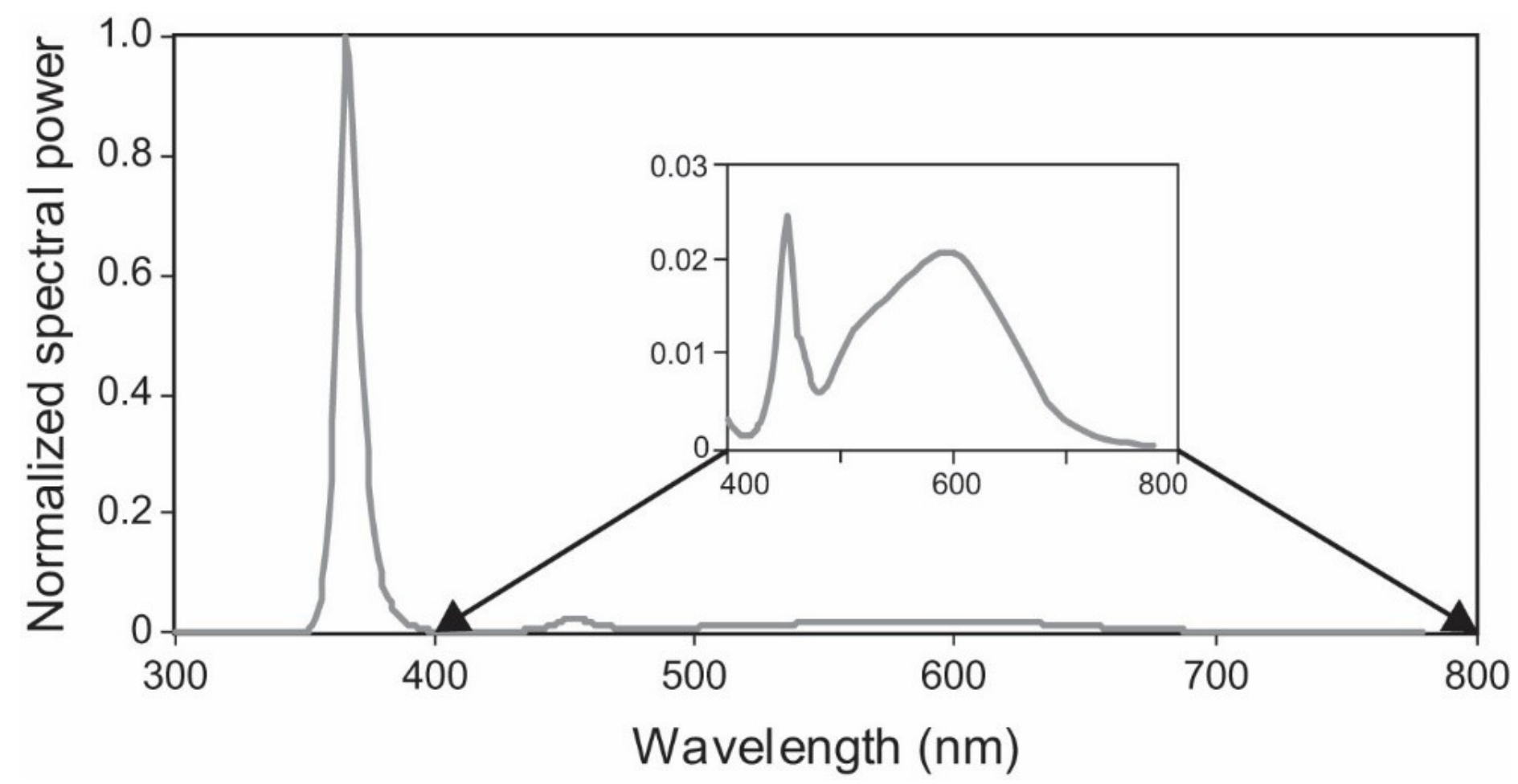

Figure 3. Relative SPDs of the two hybrid lighting LED channels, UV-A (Amax $=366 \mathrm{~nm})$ and white light

\section{Radiation Safety}

The manufacturer's recommendations for implementation safety relied on IEC standard 62471:2006, "Photobiological safety of lamps and lamp systems."(16) Standard 62471 gives thresholds for near-UV (UV-A; 315-400 nm), far-UV (actinic; 200-400 nm), and blue light $(300-700 \mathrm{~nm})$ exposures. For near UV, the irradiance limit is $10 \mathrm{~W} \mathrm{m-2}$ on the skin or at the eyes for 8 hours.
Following photometrically realistic simulations, confirmed by physical measurements, UV-A radiation emitted by the hybrid lighting system was limited to $10 \mathrm{~W} \mathrm{m-2}$ at $6 \mathrm{ft}(2 \mathrm{~m})$ above the finished floor plane, corresponding to an eye height of a very tall person. This safety limit corresponded to a UV-A irradiance level of approximately $3 \mathrm{~W} \mathrm{~m}-2$ at the counter and sink heights of $3 \mathrm{ft}(1$ $\mathrm{m}$ ) above the finished floor plane. Because Standard 62471 does not provide safety guidelines for exposures longer than 8 hours 
in a single day, the duration of UV-A operation for this study was limited to 8 continuous hours per day.

\section{Curtains}

Despite the fact that the UV-A output was set at levels deemed safe for adults,(16) extra precautions were taken to keep direct UV-A irradiance off the infant patients during the study. Weale showed, for example, that infants' crystalline lenses transmit more UV-A than older people; thus, greater protective measures were needed for this population.(18) Short, blackout-type curtains were hung in the six patient rooms (Figure 4); the bottom of these curtains was $5 \mathrm{ft} 5$ in $(1.65 \mathrm{~m})$ above the floor. In addition to curtains, nursing personnel were ordered to drape baby bassinets/incubators when occupied (Figure 4).
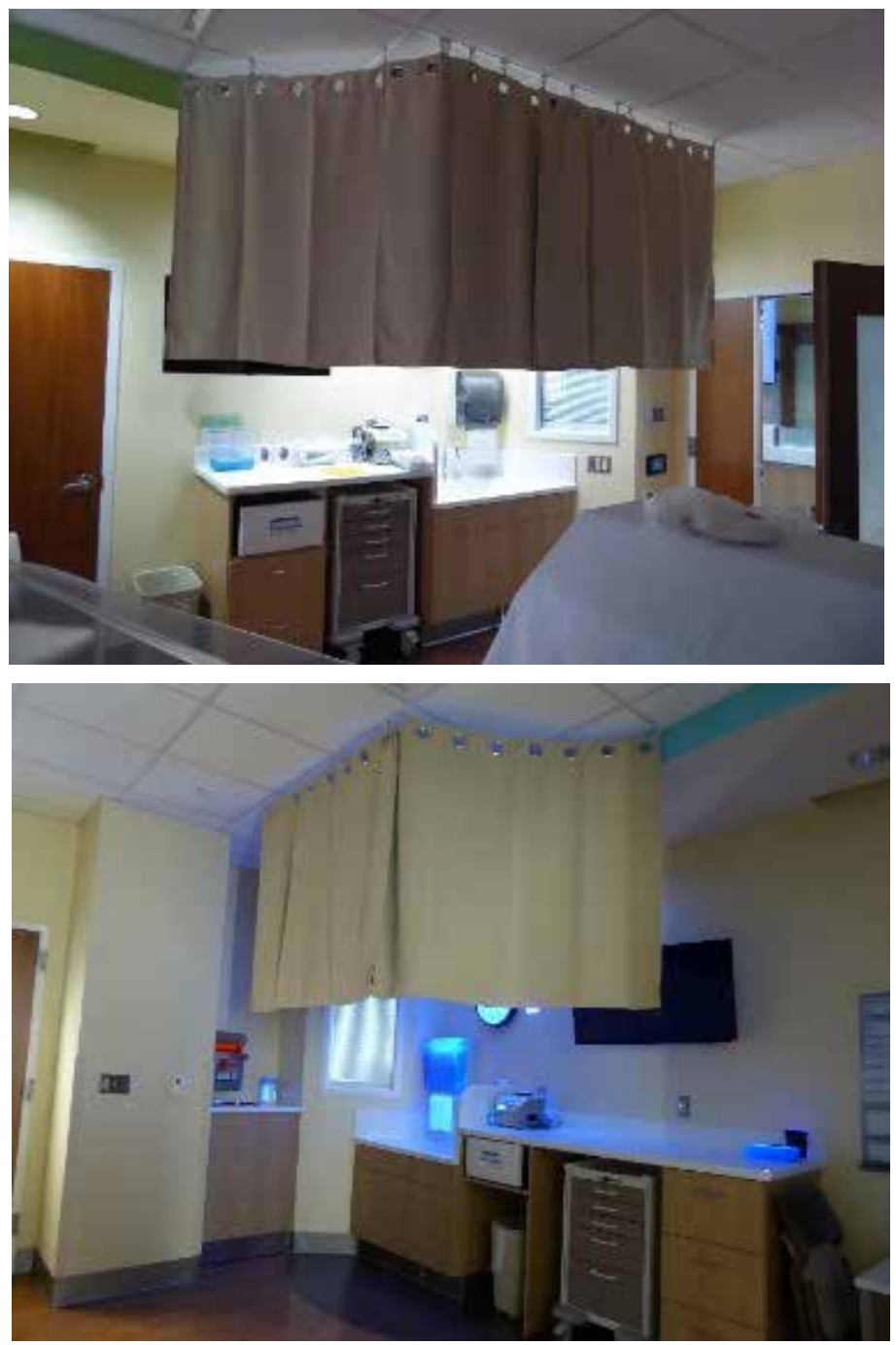

Figure 4. Examples of curtains in two of the six patient rooms; shown in foreground of left image is a baby incubator draped in fabric.

\section{Measurements}

White-light illuminance and UV-A irradiance measurements were obtained at three locations in patient rooms: the sink, the nearby counter, and the far end of the counter. These were the same locations where the adenosine triphosphate (ATP) samples were collected (see Protocol, below).
Similar white-light illuminance levels were available at the sink before and after retrofit (600-700 lx). At patient room counters, illuminance levels at full output were higher after the retrofit (800 Ix) than before the retrofit (450 lux).

As previously noted, the radiometric measurements confirmed the simulated irradiance level of $3 \mathrm{~W} \mathrm{~m}-2$ at the primary locations (sink and counter). The measurement protocol focused on these primary locations; ancillary surfaces (e.g., counter inpatient rooms) far from the hybrid luminaires naturally had lower UV-A irradiance levels. Because the infant incubators were not close to the hybrid luminaires, extensive UV-A measurements were not undertaken of incubator interiors, but as shown in Figure 5, even when the curtains were retracted, and the incubator was directly in line with the UV-A source, the fabric inside the incubator did not fluoresce like those fabrics outside the incubator. This indicates that the visually transparent cover did not transmit UV-A. Figure 5: When curtains were retracted, the fabric on the exterior of this incubator did fluoresce, but the interior did not; this suggests that the incubator transparent cover did not transmit UV-A.

\section{"When curtains were retracted, the fabric on the exterior of this incubator did fluoresce, but the interior did not; this suggests that the incubator transparent cover did not transmit UV-A."}

\section{Protocol}

A one-week protocol was repeated three times in spring 2019. The white light from the hybrid luminaires provided illumination to the counters and sinks all three weeks. The UV-A radiation from the hybrid luminaire was operated for 8 hours on Wednesdays and Thursdays each week. Adenosine triphosphate (ATP) samples were collected at sink and counter locations mornings, evenings, and midnight in three occupied patient rooms and one vacant patient room.

\section{Inoculated Culture Plates}

In a separate protocol, to directly assess the efficacy of the UV-A exposures, three pathogen types were selected for study based upon the following three criteria:

A. A pathogen previously identified as present in this NICU

B. A pathogen identified in 2014 by the Centers for Disease Control and Prevention (CDC) as among the top 10 pathogens of concern for HAls(19)

C. A pathogen identified by the NICU Director as particularly problematic

$$
\begin{gathered}
\text { Readers can also follow } \\
\text { NEONATOLOGY } \\
\text { via our Twitter Feed } \\
\text { @NEOTO }
\end{gathered}
$$




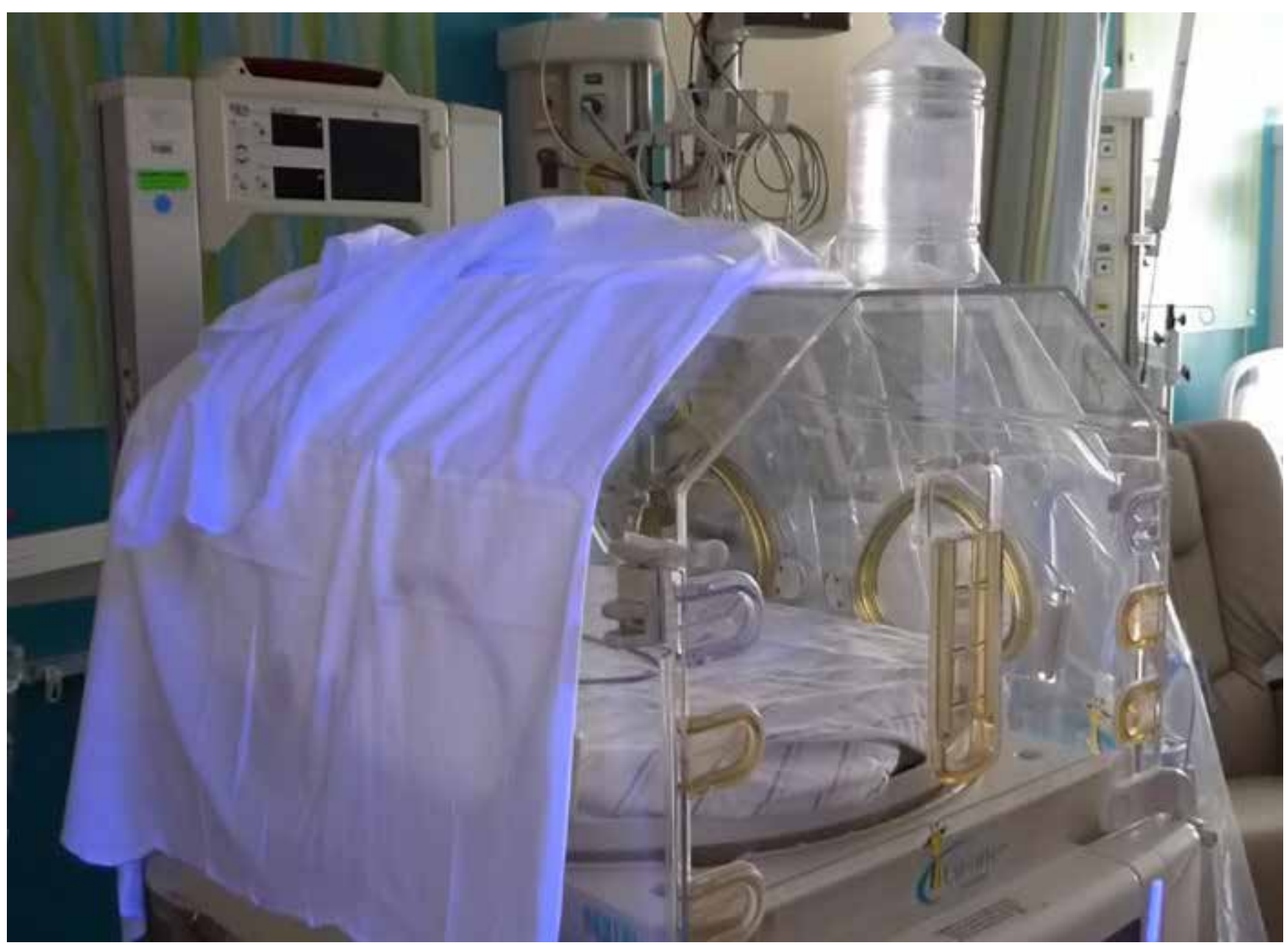

Figure 5: When curtains were retracted, the fabric on the exterior of this incubator did fluoresce, but the interior did not; this suggests that the incubator transparent cover did not transmit UV-A.

The pathogens selected for the follow-up study were:

1. Enterococcus faecalis (E. faecalis), a bacterium that causes, most commonly, urinary tract infections. This bacterium is particularly resistant to antibiotics.

2. Staphylococcus aureus (S. aureus), a bacterium associated with upper respiratory infections.(20)

3. Escherichia coli (E. coli), a diverse group of bacteria that can cause a variety of maladies, including severe dehydration.

Cultures of a given pathogen type were divided into two groups. A control group was placed on culture plates covered with a transparent, UV-blocking cover, and an intervention group was placed on culture plates covered with the usual borosilicate, UV-transparent cover plates (Figure 6). The two groups were placed at the same locations that were selected for the previous ATP sampling. The hybrid lighting system with the UV-A source energized was operated continuously for 8 hours at approximately

$3 \mathrm{~W} \mathrm{~m}-2$.

\section{Results}

\section{ATP Sampling}

ATP samples were obtained from three surfaces in 3-4 patient rooms for each of the three weeks. An analysis of the daily change in ATP counts was undertaken. After 8 hours of the UV-A application on Wednesdays there was a statistically significant reduction in ATP counts, with further, statistically significant reductions after 8 hours of UV-A application on Thursday. Importantly, on Friday, there was a statistically significant increase in ATP counts.

\section{Inoculated Culture Plates}

Student's one-tail t-tests comparing the UV-transmitting (intervention) and the UV-blocking (control) cultures showed statistically significant CFU reductions for $\mathrm{E}$. faecalis $(\mathrm{t}, 5=-1.98, \mathrm{p}=0.05)$, $\mathrm{S}$. aureus $(t, 3=-3.52, p=0.02)$ and $E$. coli. $(t, 3=-12.58, p=0.0005)$. Figure 7 shows these results in terms of percent CFU reduction; this shows the differential impact of the UV-A intervention relative to the control.

\section{Discussion}

\section{ATP Sampling}

ATP samples are routinely collected in the many units of Memorial Hospital as an inexpensive technique for quality assurance of their cleaning procedures. The hospital NICU studied here is 

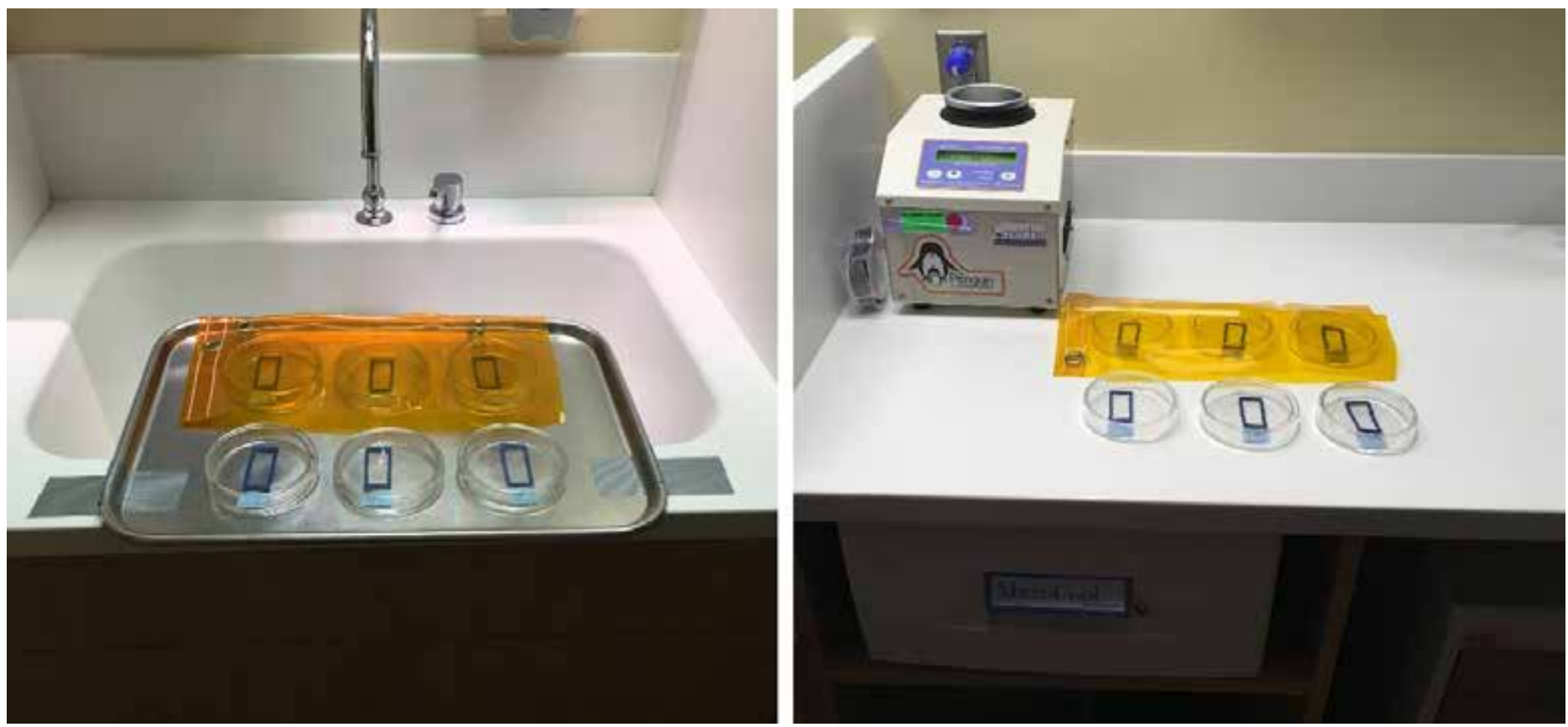

Figure 6. Inoculated culture plates at patient room sink (left) and counter (right); borosilicate glass covered all test plates, and additional orange-colored UV-blocking film covers each control group.

very clean, as reflected in the low ATP counts obtained during this study. Indeed, over the three weeks of the study, ATP counts were rarely over the threshold for cleanliness in this hospital. In fact, the NICU Director reported that his unit consistently receives internal recognition as one of the most consistently clean units in the hospital. To better gauge the level of cleanliness in the NICU, ATP spot checks of public areas in the hospital were sampled; those results supported the inference that this NICU is particularly clean.

"From an experimental perspective, low ATP counts made it difficult to assess the pathogen mitigation efficacy of the $U V-A$ radiation from the hybrid lighting system. Nevertheless, support for the effectiveness of this hybrid lighting technology for killing bacteria was obtained from an analysis of the daily change in ATP counts. Without the UV-A radiation, there was no statistically reliable change in ATP counts, but there were statistically significant reductions in ATP counts following the UV-A exposures."

From an experimental perspective, low ATP counts made it difficult to assess the pathogen mitigation efficacy of the UV-A radiation from the hybrid lighting system. Nevertheless, support for the effectiveness of this hybrid lighting technology for killing bacteria was obtained from an analysis of the daily change in ATP counts. Without the UV-A radiation, there was no statistically reliable change in ATP counts, but there were statistically significant reductions in ATP counts following the UV-A exposures. And, importantly, stopping the UV-A treatment led to a significant increase in ATP counts. After the COVID crisis abates, future demonstrations of the hybrid lighting technology are planned for hospital units with greater bio-burden. Significant reductions in pathogen counts after UV-A exposures should then be related to a reduction in $\mathrm{HAI}$ incidence.

\section{Inoculated Culture Plates}

The inoculated cell culture analysis was important for a variety of reasons. First, this NICU was particularly clean, making it difficult

\section{NATIONAL PERINATAL ASSOCIATION}

\section{Update: CORONAVIRUS}

According to data

COVID-19

published in The Lancet

PRESENTATION OF INFECTION:

clinical characteristics reported in pregnant women with confirmed COVID-19 infection are similar to those reported for non-pregnant adults.

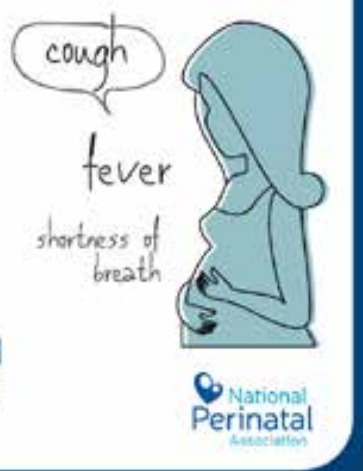

www.nationalperinatal.org 


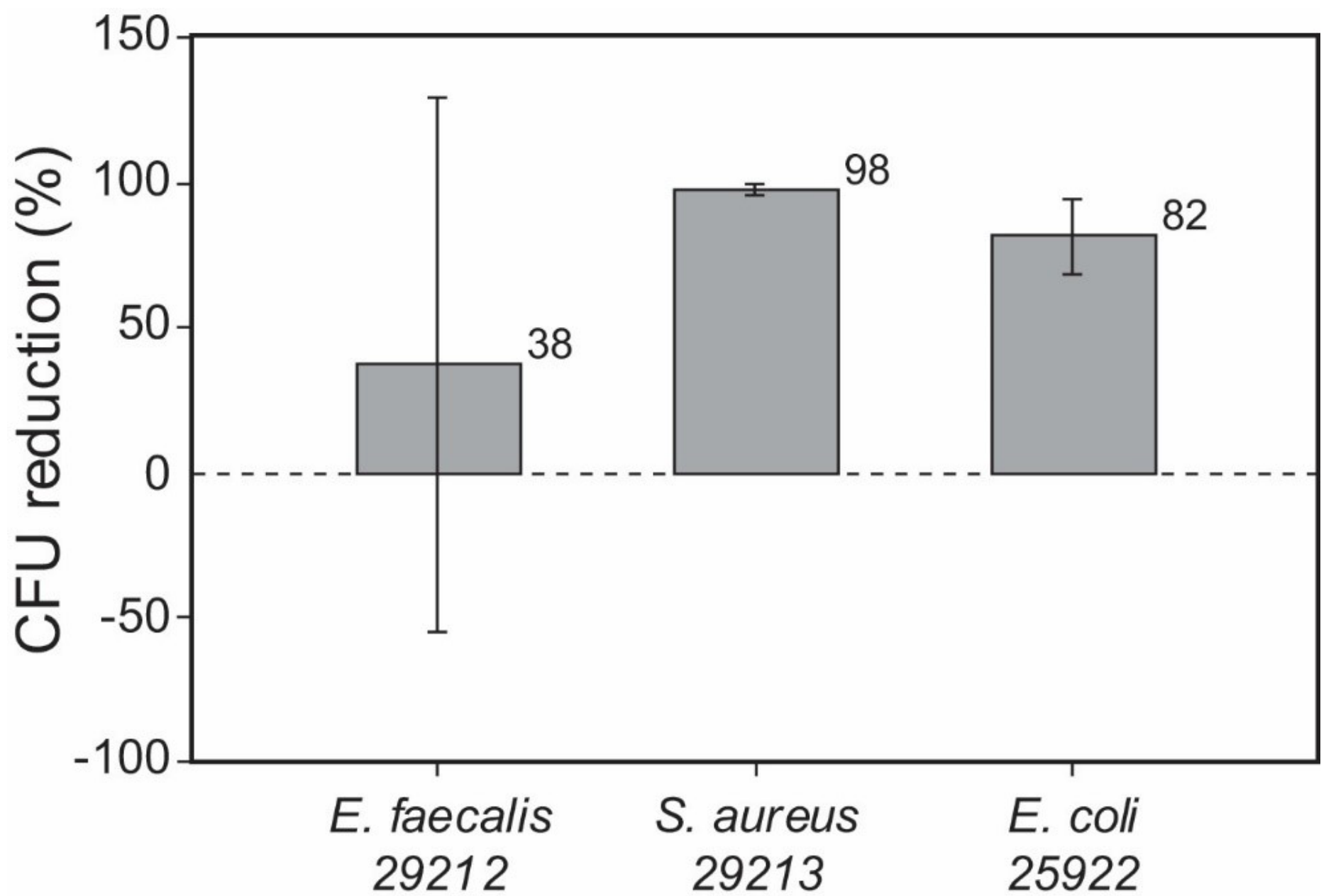

Figure 7. Average CFU percent reduction (1-(Intervention/Control)) following 8-hour exposures of $\approx 3 \mathrm{~W} m-2$ of UV-A; error bars indicate standard deviation.

to demonstrate the efficacy of the UV-A applications. Second, ATP samples do not differentiate pathogens that might cause HAls from other organic materials. Third, a side-by-side comparison of cell culture growth, ambient lighting with and without UV-A, must be conducted to demonstrate that UV-A exposures affect pathogen growth unambiguously. Specifically, the side-by-side test conducted here showed that important pathogens identified by the CDC as problematic sources of HAls and ones actually found in the NICU were directly abated by the UV-A applications actually used in the present field study.

\section{Conclusions}

The field study described here was the first to examine the efficacy of UV-A for reducing pathogens in the context of a working hospital. The hybrid lighting system used in the present study could independently emit visible white light, UV-A radiation, or both. A series of analyses support the inference that the UV-A radiation will reduce the burden of HAls in doses set to minimize negative health effects for adult occupants ( $\max =10 \mathrm{~W} \mathrm{m-2}$ for 8 hours). The hybrid lighting system is safe to operate in occupied spaces under the radiation restrictions described here, but long-term and collateral effects on materials, and people need to be carefully tested before it should be widely adopted.

\section{References:}

1. Weber DJ, Rutala WA, Anderson DJ, Chen LF, Sickbert- devices and hydrogen peroxide systems for terminal room decontamination: focus on clinical trials. Am J Infect Control. 2016;44(5):e77-e84.

2. Carling P. Methods for assessing the adequacy of practice and improving room disinfection. Am J Infect Control. 2013;41(5):S20-S5.

3. Shaughnessy MK, Micielli RL, DePestel DD, Arndt J, Strachan CL, Welch KB, et al. Evaluation of hospital room assignment and acquisition of Clostridium difficile infection. Infect Control Hosp Epidemiol. 2011;32(3):201-6.

4. Dancer SJ. Controlling hospital-acquired infection: focus on the role of the environment and new technologies for decontamination. Clin Microbiol Rev. 2014;27(4):665-90.

5. Han JH, Sullivan N, Leas BF, Pegues DA, Kaczmarek JL, Umscheid CA. Cleaning hospital room surfaces to prevent health care-associated infections: a technical brief. Ann Intern Med. 2015;163(8):598-607.

6. Boyce JM, Havill NL. Evaluation of a new hydrogen peroxide wipe disinfectant. Infect Control Hosp Epidemiol. 2013;34(5):521-3.

7. Boyce JM. Modern technologies for improving cleaning and disinfection of environmental surfaces in hospitals. Antimicrobial Resistance \& Infection Control. 2016;5(1):10.

8. Kovach CR, Taneli Y, Neiman T, Dyer EM, Arzaga AJA, Kelber ST. Evaluation of an ultraviolet room disinfection protocol to decrease nursing home microbial burden, infection and hospitalization rates. BMC Infect Dis. 2017;17(1):186. 
9. Rutala WA, Weber DJ. Best practices for disinfection of noncritical environmental surfaces and equipment in health care facilities: A bundle approach. Am J Infect Control. 2019;47:A96-A105.

10. Livingston SH, Cadnum JL, Benner KJ, Donskey CJ. Efficacy of an ultraviolet-A lighting system for continuous decontamination of health care-associated pathogens on surfaces. Am J Infect Control. 2019.

11. Tseng C-C, Li C-S. Inactivation of virus-containing aerosols by ultraviolet germicidal irradiation. Aerosol Science and Technology. 2005;39(12):1136-42.

12. Health Quality Ontario. Portable Ultraviolet Light SurfaceDisinfecting Devices for Prevention of Hospital-Acquired Infections: A Health Technology Assessment. Ont Health Technol Assess Ser. 2018;18(1):1-73. PubMed PMID: 29487629.

13. Wong J, Parisi A. Assessment of ultraviolet radiation exposures in photobiological experiments. Proceedings of the Second Internet Conference on Photochemistry and Photobiology. 1999:1-19.

14. Bullough JD. The blue-light hazard: A review. JIES. 2000;29. doi: 10.1080/00994480.2000.10748312.

15. Bullough JD, Bierman A, Rea MS. Evaluating the bluelight hazard from solid state lighting. Int J Occup Saf Ergonomics. 2017:10.1080/10803548.2017.1375172. doi: 10.1080/10803548.2017.1375172.

16. International Electrotechnical Commission. IEC 62471:2006 Photobiological safety of lamps and lamp systems. International Standard. 2006.

17. Brons J, Bierman A, White R, Benner K, Deng L, Rea M. An assessment of a hybrid lighting system that employs ultraviolet-A for mitigating healthcare-associated infections in a newborn intensive care unit. Light Res Technol. 2020:1477153520904107.

18. Weale R. Age and the transmittance of the human crystalline lens. J Physiol. 1988;395(1):577-87.

19. Magill SS, Edwards JR, Bamberg W, Beldavs ZG, Dumyati $G$, Kainer MA, et al. Multistate point-prevalence survey of health care-associated infections. N Engl J Med. 2014;370(13):1198-208.

20. Rana D, Abughali N, Kumar D, Super DM, Jacobs MR, Kumar ML. Staphylococcus aureus, including community-acquired methicillin-resistant S. aureus, in a level III NICU: 2001 to 2008. Am J Perinatol. 2012;29(6):401-8. Epub 2012/03/09. doi: 10.1055/s-0032-1304819. PubMed PMID: 22399220.

Declaration of conflicting interests

The authors declared no potential conflicts of interest with respect to the research, authorship, and/or publication of this article.

\section{Funding}

This study was funded by GE Current, a Daintree company.

\section{Acknowledgments}

The authors would like to thank GE Current, a Daintree company, for sponsoring this research; Jane Kaiser and Mashelle Monhaut from the Memorial Hospital NICU for supporting this research; Rebekah Mullaney from the Lighting Research Center for assistance with preparing the manuscript.

\section{NT}
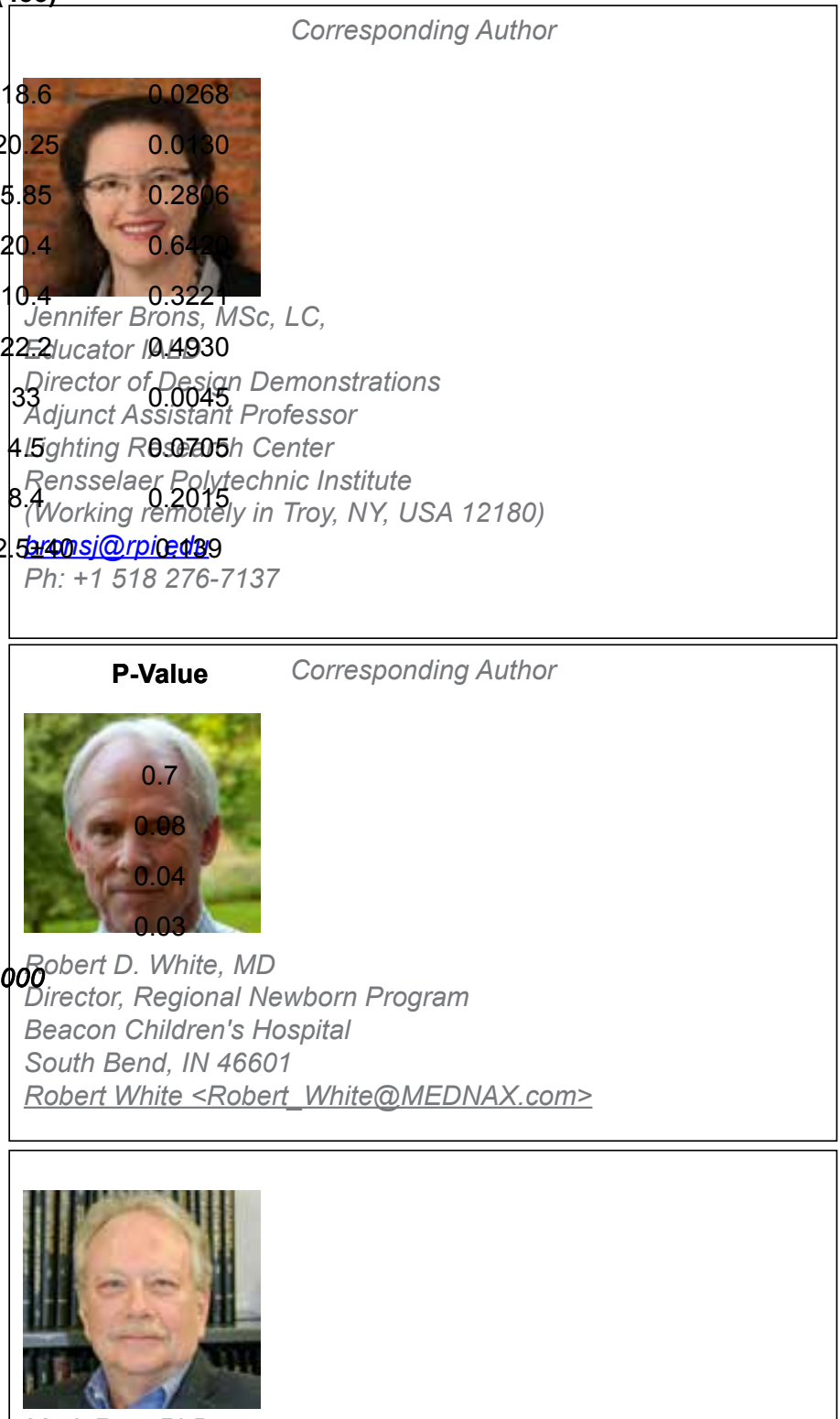

Mark Rea, PhD

Professor

Lighting Research Center

Rensselaer Polytechnic Institute

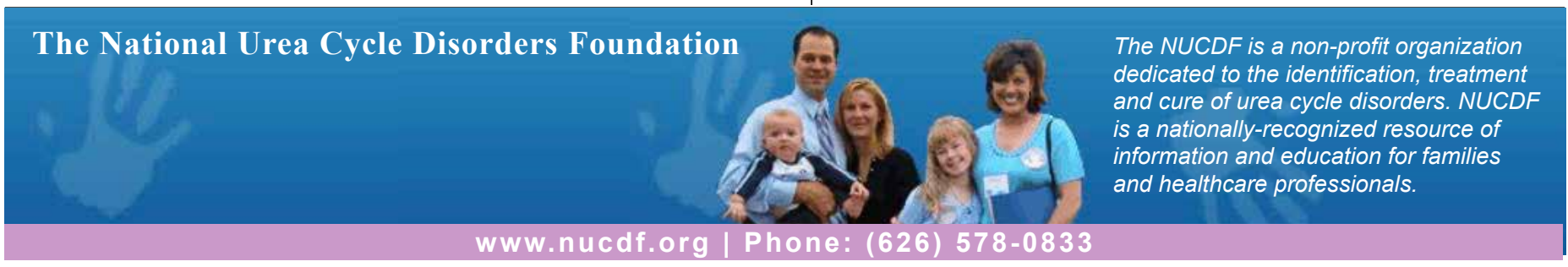

